\title{
Improved inter-device variability in graphene liquid gate sensors by laser treatment
}

\author{
Jorge Ávila*, Jose Galdon*, Norberto Salazar, Maria-Isabel Recio, Carlos Navarro, Carlos Marquez and Francisco Gamiz \\ Nanoelectronics Research Group (CITIC-UGR), Department of Electronics, \\ University of Granada, 18071, Granada, Spain \\ *These authors contributed equally to this work. \\ jorgeavila@ugr.es, jcgaldon@ugr.es
}

\begin{abstract}
We investigate the influence of a visible laser treatment on the electrical performance of CVD-grown graphenebased liquid gate sensors. This method allows to treat locally the graphene sheet, improving the electrical characteristic for biochemical sensing applications. Optimizing the laser exposure, the Dirac point (minimum conductivity voltage) was shifted around $300 \mathrm{mV}$ to lower voltages, together with a decrease of the inter-device electrical variability. These results open the door to use the laser treatment to increase the sensibility and reproducibility of liquid gate graphene-based devices as sensors or biosensors.
\end{abstract}

\section{INTRODUCTION}

Graphene, a monolayer of carbon atoms packed into a twodimensional honeycomb lattice, has received an exceptional attention due to its excellent electrical, optical, thermal and bendability properties [1]-[3]. In addition, graphene is very sensitive to changes in the surroundings while its reduced thickness improves the electrostatic control of the channel, thus making it particularly attractive for sensor applications [4]. However, in the case of biosensors, they should operate at or near physiological conditions, so it is essential to use graphene in aqueous solutions. Electrochemical studies have shown that the ions accumulate at the surface of graphene when a gate voltage is applied between the electrodes, without charge transferred across the interface [5]. These studies suggest that graphene operates nearly as an ideal polarizable electrode [6], [7]. The graphene-electrolyte interface is typically modeled as an electrical double layer capacitance (EDLC), constituted by two layers of ions that are created at the surface of graphene. The first layer is composed of ions of opposite charges to those present in the graphene, and the second layer is composed of positive and negative charged ions that progressively reach the potential of the solution far from the graphene surface. The EDLC can be modulated applying a voltage at a reference electrode immersed in the electrolyte solution, controlling the number of free carriers in graphene, and therefore its conductivity. The minimum of free carriers, and thus the minimum of conductivity, is reached when the valence and the conduction band meet at a point called the Dirac point, the gate bias at which the Fermi level reaches the Dirac point is the charge neutrality point or Dirac voltage.

Electrochemical gated graphene sensors employing electrolytes such as ionic liquids and aqueous solutions have been extensively reported, showing excellent performances [8], [9]. Nonetheless, due to the high sensitivity of graphene and the complex nature of the electrolyte solutions, the reproducibility between devices can be challenging. For example, some factors that affect the inter-device reproducibility are the different doping levels of the graphene sheet due to graphene-substrate interactions or fabrication residues [10]. Moreover, common reliability disturbances such as interface states, traps and mobile charges also affect the sensing capability of the fabricated devices. Different strategies have been explored in order to clean of residues and improve the quality of the graphene sheet, as thermal annealing, plasma, UV-Vis light, electrical or mechanical treatments [11]. In this work, we have explored the ablation of the graphene layer using a visible laser as a straightforward and rapid treatment to improve the inter-device electrical variability in graphene-based liquid gate sensors. This strategy can modify locally the graphene surface without compromising the rest of the device, being useful if sensible materials are presented and to avoid damaging the contacts. Employing this method, we have observed a reduced Dirac voltage and an improved inter-device electrical variability in graphene-based sensors.

\section{EXPERIMENTAL SETUP}

Graphene was synthesized through low-pressure chemical vapor deposition (LPCVD) and polycrystalline copper foil was used as catalytic substrate. The reaction was made at 1 Torr using methane $\left(\mathrm{CH}_{4}\right)$ as a carbon precursor. The foils were first heated up to $1000^{\circ} \mathrm{C}$ in a $\mathrm{H}_{2}$ environment to reduce the native copper oxide on the copper foil surface. Then, a $\mathrm{H}_{2} / \mathrm{CH}_{4}$ gas mixture (50 $\mathrm{sccm}$ : $30 \mathrm{sccm}$ ) was added during the graphene growth at $1000{ }^{\circ} \mathrm{C}$ for 30 minutes. The cooling down step was made by opening the furnace, during this step the gas composition was remained the same. Then, graphene layers were transferred to cleaned quartz substrates using the PMMA based technique [12]. The device processing is illustrated in Figure 1: Initially, reactive-ion etching (RIE) at $10 \mathrm{~W}$ and $30 \mathrm{sccm}$ of $\mathrm{O}_{2}$ was used to pattern the graphene employing a solid mask. Up to six sensors were fabricated on each substrate. Then, the $\mathrm{Cr}(5 \mathrm{~nm}) / \mathrm{Au}(100 \mathrm{~nm})$ electrodes were deposited by physical vapor deposition. Finally, the laser treatment was performed on the graphene layer using a computer- 
controlled laser engraver (Laserbot from Makeblock). The laser wavelength was $445 \mathrm{~nm}$, and the power was set to $25 \%$ with respect to the maximum power $(1600 \mathrm{~mW})$. To evaluate the electrochemical characteristics of the devices, a phosphate buffered saline solution (PBS) at a $1 \mathrm{X}$ concentration, was placed covering the graphene to perform the liquid gate. PBS is a buffer solution commonly used in biochemical research, as the osmolarity and ion concentrations of the solutions match those of the human body. Hence, using this buffer solution, we evaluated the sensor in near human physiological conditions. The static DC characteristics were acquired using a Keithley SCS 4200 and an Agilent B1500 systems. The low-frequency noise characterization was carried out using a low-noise-current amplifier connected to a software-based spectrum analyzer [13].

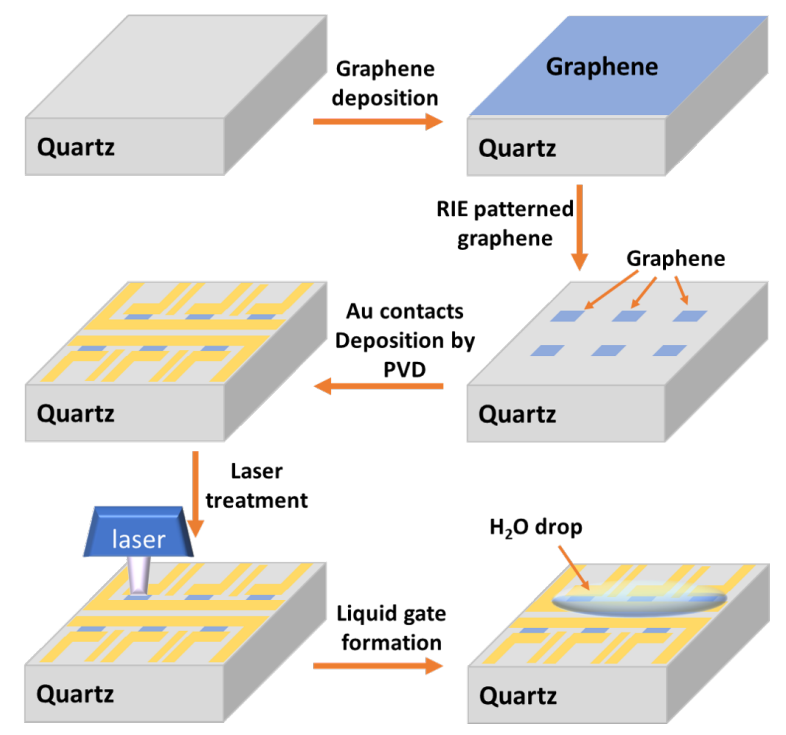

Fig. 1. Scheme of the fabrication flow of the liquid gate graphene devices including the laser treatment.

\section{RESUlTS AND DISCUSSION}

Before considering the double layer capacitance effect in the devices, the laser radiation on the graphene sheet has been evaluated. The graphene layer resistance was measured, without a liquid gate, at a fixed drain-source voltage $\left(V_{D}=0.1 \mathrm{~V}\right)$ before and after each laser exposure (Figure 2.a). An increase in the resistance of the device was observed following an exponential trend with the number of laser exposure cycles. The resistance value saturated around $2.8 \mathrm{k} \Omega$ after 6 to 8 cycles. In order to evaluate the structural repercussion of the laser ablation and determine the origin of the increased electrical resistance of the graphene, Raman spectroscopy analysis was carried out before and after the laser treatment (Figure 2.b). Prior to laser exposure, the Raman spectrum of the graphene device shows the signature for pristine singlelayer graphene, with a $\mathrm{G}$ peak at $\approx 1590 \mathrm{~cm}^{-1}$ and a $2 \mathrm{D}$ peak at $\approx 2680 \mathrm{~cm}^{-1}$. After the laser treatment, an increase of the $\mathrm{D}$ peak $\left(\approx 1340 \mathrm{~cm}^{-1}\right)$, as well as the emergence of the D' peak $\left(\approx 1620 \mathrm{~cm}^{-1}\right)$ can be observed.

These results corroborate that the laser treatment has an effect on the structural order of the graphene. The emergence of the D' peak could be related to the formation of $\mathrm{sp}^{3}$-type defects [14], [15]. These new defects have an adverse impact on the electrical behavior, increasing the graphene resistance. Nevertheless, the resistance stabilized at higher number of laser treatments, indicating that possibly the effect of the laser in the graphene structure saturates. A possible explanation is that these defects are formed mainly in the grain boundaries, where dangling bonds are receiving enough energy to react with the ambient atmosphere, generating some oxide species [16]. These species would hinder the electric transport between grains, increasing the electric resistance. Despite deteriorating the sheet resistance of the graphene layers, these defects may have different implications when the graphene-electrolyte interface is formed.
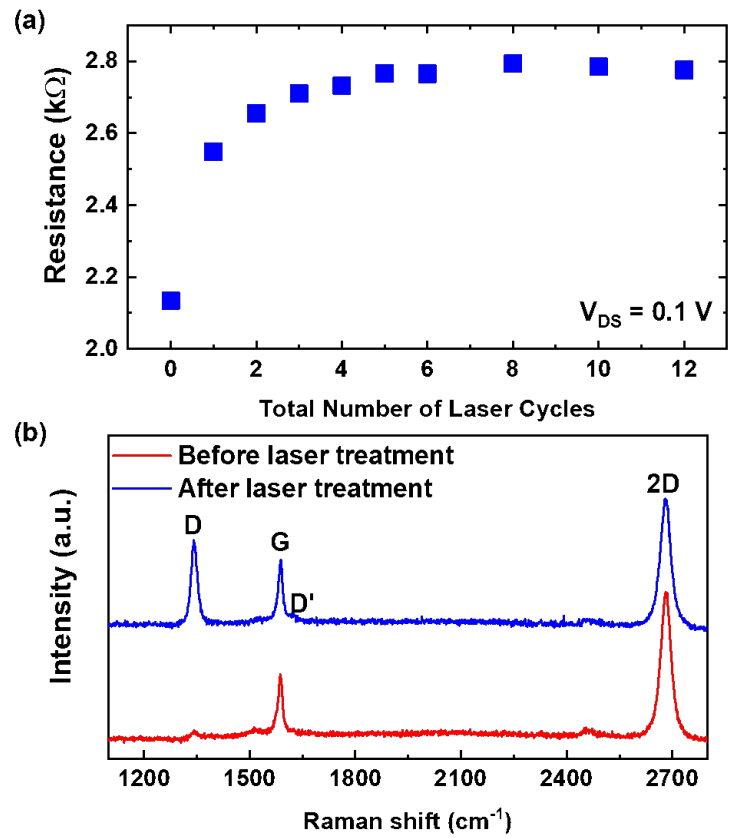

Fig. 2. a) The graphene device resistance measured at $0.1 \mathrm{~V}$ drain-source bias without liquid gate for different numbers of laser cycles and, b) the graphene Raman spectra before and after the laser treatment. The Raman laser wavelength is $532 \mathrm{~nm}$.

After the deposition of the liquid gate and the formation of the electrical double layer capacitance, we measured the transfer characteristic curve $\left(R=V_{D} / I_{D}\right)$ of a device using two different metals as the gate electrode (Figure 3 ). A proper modulation of the resistance as a function of the liquid gate voltage is observed, together with a shift of the Dirac point depending on the metal electrode. This shift from $0.83 \mathrm{~V}$ (gold) to $0.27 \mathrm{~V}$ (silver) is mainly due to the difference in the work function of the employed metals. In order to work in aqueous solutions, low voltages are desirable to keep the working conditions below the water electrolysis potential 
$(1.2 \mathrm{~V})$, avoiding secondary reactions, keeping a low gatedrain current [17]. The lower Dirac voltage for the silver gate electrode can be interesting for low energy consumption applications. However, silver can easily be oxidized in contact with the aqueous solution. Hence, it is preferable the use of a gold electrode as a more electrochemical stable option despite the higher voltages. In the following devices, gold was used as the gate electrode.

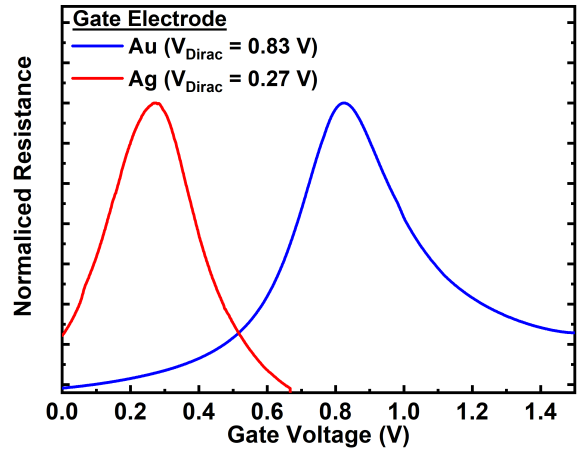

Fig. 3. Resistance versus gate voltage characteristic curves for a device measured employing gold(blue line) or silver (red line) as the gate electrode. The drain source bias was set at $0.1 \mathrm{~V}$. The Dirac voltage is defined as the voltage when the resistance reaches the maximum.

Figure 4.a shows the transfer characteristic curves of a device when using the liquid gate for successive laser treatment cycles. Note that in all the cases, there is a modulation of the channel conductivity as a function of the liquid gate voltage. As observed, the device resistance initially decreases with the successive laser cycles, but after 4 cycles it starts to saturate. At the same time, the Dirac point shifts to lower voltages from $1 \mathrm{~V}$ before the laser treatment, down to $0.66 \mathrm{~V}$ after 8 cycles (inset in Figure 4.a). Then, at higher number of laser cycles, the rise of the resistance is accelerated and the Dirac voltage shifts to higher voltages. This indicates that a high number of laser cycles is drastically damaging the sensor. The increase in the resistance is in agreement with the Raman interpretation, indicating that after the laser exposure the graphene conductivity is hindered, harming the electrical performance. However, at low number of laser cycles, the sensing capabilities are improved with a lower resistance and reduced Dirac voltages compared to the device without laser treatment. When comparing the Dirac voltages of several devices before and after the laser treatment (for a total number of 4 laser cycles) a significant reduction of the electrical variability among devices is observed (Figure 4.b). The laser treatment consisting of 4 cycles reduces the standard deviation from 65 to $24 \mathrm{mV}$, together with an improved average Dirac voltage of $810 \mathrm{mV}$. Note that the inter-device variability is a critical constraint for sensing applications. A possible explanation of the variability improvement observed could reside in a laser-induced transformation of the graphene structure. Induced defects and oxidized species at grain boundaries can improve the electrical double layer capacitance, which finally improves the liquid gate/interface, making it more reliable for sensing and less variable among devices [18], [19].
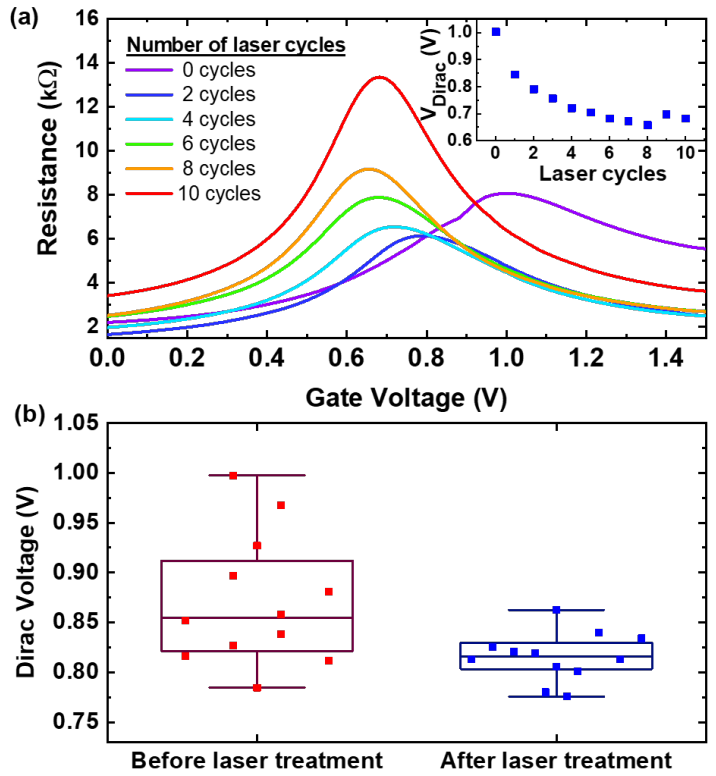

Fig. 4. a) Resistance versus gate voltage characteristic curves for a device measured at different number of laser cycles. The drain source bias was set at $0.1 \mathrm{~V}$. The Dirac voltage is defined as the voltage when the resistance reaches the maximum. b) Dirac voltages distribution of 16 devices measured before and after a laser treatment of 4 cycles.

Low-frequency noise characterization sheds light on the defect implication in the graphene electrical performance after the laser ablation. As figure 5 shows, the graphene layer presents a normalized power spectral density (PSD) of the noise formed by a flicker or 1decade/1decade (1/f) contribution at low frequency and a Lorentzian contribution with center frequency around $10^{4} \mathrm{~Hz}$. This result indicates that graphene sheets are affected by carrier number fluctuations at low frequencies and by capture and emission processes of carriers at higher frequencies [20]. According to the similar spectrum observed before and after the laser irradiation (even lightly lower), these fluctuations are not enhanced by the laser treatment, suggesting a graphene resistance increased without a degradation of the interface after the laser exposure. This result is in agreement with the lower variability among devices observed in Figure 4.b after the laser irradiation.

\section{CONCLUSION}

Despite presenting a slight increase in the graphene resistance after laser exposure, the graphene-based liquid gate sensors show a reduced Dirac point and inter-device variability. These results indicate that there is a trade-off between some grade of graphene degradation and an improvement at the liquid gate/graphene interface. Moreover, the laser exposure does not affect the power spectral density of the current 


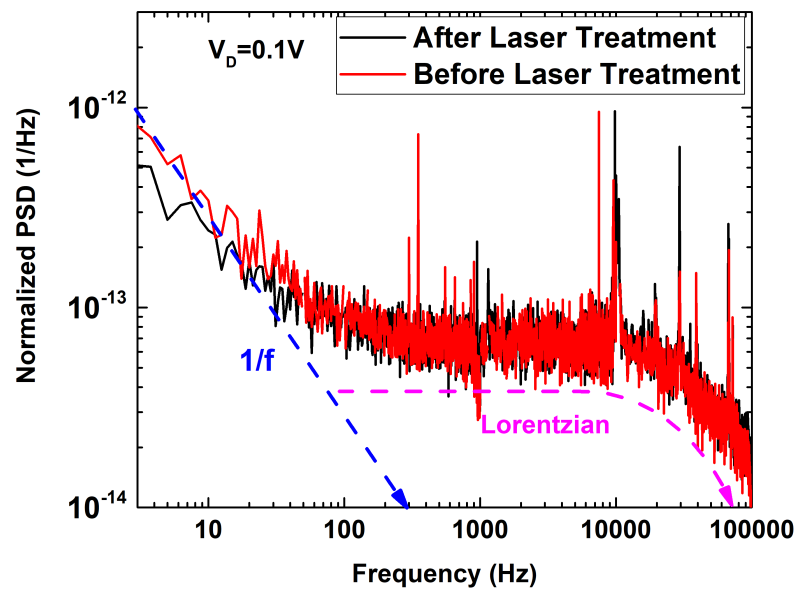

Fig. 5. Normalized power spectral density of the noise for a device before and after the laser treatment.

discarding an interface degradation. Finding the origin of this phenomenon seems critical to optimize the device performance for sensing using liquid gates. Hence, the use of the laser treatment could be an advantageous technique to increase the sensibility and reproducibility of these graphene-based devices for applications as sensors or biosensors.

\section{ACKNOWLEDGMENT}

This work has received funding from the European Union's Horizon 2020 research and innovation programme under the Marie Skłodowska-Curie grant agreement No 895322, from the Spanish Program (TEC2017-89800-R). SUPERA COVID19 Fund and CRUE-Santander, Regional Program FEDER UGRVID (CV20-36685), P18-RT-4826 project and UGRMADOC CEMIX 2D-EDEX are also thanked for financial support.

\section{REFERENCES}

[1] K. S. Novoselov, A. K. Geim, S. V. Morozov, D. Jiang, Y. Zhang, S. V. Dubonos, I. V. Grigorieva, and A. A. Firsov, "Electric Field Effect in Atomically Thin Carbon Films," Science, vol. 306, no. 5696, pp. 666-9, oct 2004.

[2] A. K. Geim and K. S. Novoselov, "The rise of graphene." Nature materials, vol. 6, no. 3, pp. 183-191, 2007. [Online]. Available: http://www.nature.com/doifinder/10.1038/nmat1849

[3] K. S. Novoselov, V. I. Fal ko, L. Colombo, P. R. Gellert, M. G. Schwab, and K. Kim, "A roadmap for graphene," Nature, vol. 490, no. 7419, pp. 192-200, 2012.

[4] C. W. Lee, J. M. Suh, and H. W. Jang, "Chemical Sensors Based on Two-Dimensional (2D) Materials for Selective Detection of Ions and Molecules in Liquid," Frontiers in Chemistry, vol. 7, no. November, pp. 1-21, nov 2019. [Online]. Available: https://www.frontiersin.org/article/10.3389/fchem.2019.00708/full

[5] A. T. Valota, I. A. Kinloch, K. S. Novoselov, C. Casiraghi, A. Eckmann, E. W. Hill, and R. A. W. Dryfe, "Electrochemical Behavior of Monolayer and Bilayer Graphene," ACS Nano, vol. 5, no. 11, pp. 8809-8815, nov 2011. [Online]. Available: https://pubs.acs.org/doi/10.1021/nn202878f
[6] M. Dankerl, M. V. Hauf, A. Lippert, L. H. Hess, S. Birner, I. D. Sharp, A. Mahmood, P. Mallet, J.-Y. Veuillen, M. Stutzmann, and J. A. Garrido, "Graphene Solution-Gated Field-Effect Transistor Array for Sensing Applications," Advanced Functional Materials, vol. 20, no. 18, pp. 3117-3124, sep 2010. [Online]. Available: https://onlinelibrary.wiley.com/doi/10.1002/adfm.201000724

[7] X. Du, H. Guo, Y. Jin, Q. Jin, and J. Zhao, "Electrochemistry Investigation on the Graphene/Electrolyte Interface," Electroanalysis, vol. 27, no. 12, pp. 2760-2765, dec 2015. [Online]. Available: https://onlinelibrary.wiley.com/doi/10.1002/elan.201500302

[8] F. Chen, Q. Qing, J. Xia, J. Li, and N. Tao, "Electrochemical gatecontrolled charge transport in graphene in ionic liquid and aqueous solution," Journal of the American Chemical Society, vol. 131, no. 29, pp. 9908-9909, 2009.

[9] N. Liu, R. Chen, and Q. Wan, "Recent advances in electric-double-layer transistors for bio-chemical sensing applications," Sensors (Switzerland), vol. 19, no. 15, 2019.

[10] A. Pirkle, J. Chan, A. Venugopal, D. Hinojos, C. W. Magnuson, S. McDonnell, L. Colombo, E. M. Vogel, R. S. Ruoff, and R. M. Wallace, "The effect of chemical residues on the physical and electrical properties of chemical vapor deposited graphene transferred to $\mathrm{SiO} 2$," Applied Physics Letters, vol. 99, no. 12, p. 122108, sep 2011. [Online]. Available: http://aip.scitation.org/doi/10.1063/1.3643444

[11] B. Zhuang, S. Li, S. Li, and J. Yin, "Ways to eliminate PMMA residues on graphene superclean graphene," Carbon, vol. 173, pp. 609-636, mar 2021. [Online]. Available: https://linkinghub.elsevier.com/retrieve/pii/S000862232031126X

[12] G. Borin Barin, Y. Song, I. de Fátima Gimenez, A. G. Souza Filho, L. S. Barreto, and J. Kong, "Optimized graphene transfer: Influence of polymethylmethacrylate (PMMA) layer concentration and baking time on graphene final performance," Carbon, vol. 84, no. C, pp. 82-90, apr 2015. [Online]. Available: https://linkinghub.elsevier.com/retrieve/pii/S0008622314011269

[13] J. Chroboczek, "Automatic, wafer-level, low frequency noise measurements for the interface slow trap density evaluation," in International Conference on Microelectronic Test Structures, 2003. IEEE, 2003, pp. 95-98. [Online]. Available: 10.1109/ICMTS.2003.1197409

[14] A. Eckmann, A. Felten, A. Mishchenko, L. Britnell, R. Krupke, K. S. Novoselov, and C. Casiraghi, "Probing the Nature of Defects in Graphene by Raman Spectroscopy," Nano Letters, vol. 12, no. 8, pp. 3925-3930, aug 2012. [Online]. Available: https://pubs.acs.org/doi/10.1021/nl300901a

[15] T. Huang, J. Long, M. Zhong, J. Jiang, X. Ye, Z. Lin, and L. Li, "The effects of low power density co2 laser irradiation on graphene properties," Applied surface science, vol. 273, pp. 502-506, 2013.

[16] D. L. Duong, G. H. Han, S. M. Lee, F. Gunes, E. S. Kim, S. T. Kim, H. Kim, Q. H. Ta, K. P. So, S. J. Yoon, S. J. Chae, Y. W. Jo, M. H. Park, S. H. Chae, S. C. Lim, J. Y. Choi, and Y. H. Lee, "Probing graphene grain boundaries with optical microscopy," Nature, vol. 490, no. 7419, pp. 235-239, 2012. [Online]. Available: http://dx.doi.org/10.1038/nature 11562

[17] H. Ohta, Y. Sato, T. Kato, S. Kim, K. Nomura, Y. Ikuhara, and H. Hosono, "Field-induced water electrolysis switches an oxide semiconductor from an insulator to a metal," Nature Communications, vol. 1, no. 8,2010

[18] B. Standley, A. Mendez, E. Schmidgall, and M. Bockrath, "Graphene-Graphite Oxide Field-Effect Transistors," Nano Letters, vol. 12, no. 3, pp. 1165-1169, mar 2012. [Online]. Available: https://pubs.acs.org/doi/10.1021/nl2028415

[19] Y. Yang and R. Murali, "Binding mechanisms of molecular oxygen and moisture to graphene," Applied Physics Letters, vol. 98, no. 9, p. 093116, feb 2011. [Online]. Available: http://aip.scitation.org/doi/10.1063/1.3562317

[20] T. A. Oproglidis, T. A. Karatsori, C. G. Theodorou, D. Tassis, S. Barraud, G. Ghibaudo, and C. A. Dimitriadis, "Origin of Low-Frequency Noise in Triple-Gate Junctionless nMOSFETs," IEEE Transactions on Electron Devices, vol. 65, no. 12, pp. 5481-5486, dec 2018. [Online]. Available: https://ieeexplore.ieee.org/document/8494709/ 